ARTICLE

\title{
Semiconductor to metal transition in two- dimensional gold and its van der Waals heterostack with graphene
}

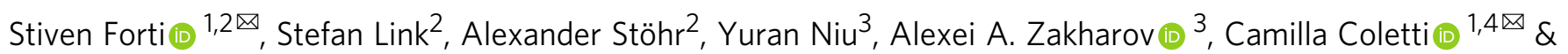
Ulrich Starke (i) ${ }^{2}$

The synthesis of two-dimensional (2D) transition metals has attracted growing attention for both fundamental and application-oriented investigations, such as 2D magnetism, nanoplasmonics and non-linear optics. However, the large-area synthesis of this class of materials in a single-layer form poses non-trivial difficulties. Here we present the synthesis of a largearea 2D gold layer, stabilized in between silicon carbide and monolayer graphene. We show that the $2 \mathrm{D}-\mathrm{Au} \mathrm{ML}$ is a semiconductor with the valence band maximum $50 \mathrm{meV}$ below the Fermi level. The graphene and gold layers are largely non-interacting, thereby defining a class of van der Waals heterostructure. The 2D-Au bands, exhibit a $225 \mathrm{meV}$ spin-orbit splitting along the $\overline{\Gamma K}$ direction, making it appealing for spin-related applications. By tuning the amount of gold at the $\mathrm{SiC} /$ graphene interface, we induce a semiconductor to metal transition in the 2D-Au, which has not yet been observed and hosts great interest for fundamental physics.

\footnotetext{
${ }^{1}$ Center for Nanotechnology Innovation @ NEST, Istituto Italiano di Tecnologia, Piazza San Silvestro 12, 56127 Pisa, Italy. ${ }^{2}$ Max-Planck-Institut für Festkörperforschung, Heisenbergstr. 1, D-70569 Stuttgart, Germany. ${ }^{3}$ MAX IV Laboratory, Lund University, P.O. Box 118, Lund S-22100, Sweden. ${ }^{4}$ Graphene Labs, Istituto Italiano di Tecnologia, via Morego 30, 16163 Genova, Italy. ${ }_{\text {email: stiven.forti@iit.it; camilla.coletti@iit.it }}$
} 
owering the dimensionality of a given compound has become a big hype in the field of material research, as this opens the possibility to explore and exploit properties, which are inaccessible in a bulk crystal. While several compounds can be relatively easily rendered two-dimensional (2D) with physical approaches $^{1-13}$, many others are difficult to isolate. In particular, there are essentially no single-element materials other than graphite that can be easily exfoliated ${ }^{14}$. 2D metals see a vast field for applications, ranging from catalysis to sensing ${ }^{15,16}$, passing through enhanced magnetism ${ }^{17-20}$. This enticing applicative potential has prompted the development of various approaches for the synthesis of low-dimensional metals ${ }^{15}$. Several chemistrybased strategies have been implemented, however mostly yielding nanostructures of different shapes and geometries ${ }^{15}$. Physical methods have been shown to effectively yield monolayers of transition metals, typically on other metal surfaces ${ }^{21-23}$. The main focus of those types of investigations was the interactions between heavy atoms yielding large spin-orbit splitting of surface states, which might be useful for spintronic devices ${ }^{24}$. However, the strong interaction between the metallic substrate and the 2D layer typically hinders the possibility to access the intrinsic properties of the latter. Also, keeping the surface energy low enough to avoid the growth of multilayer islands is a non-trivial problem to address and often a capping medium solution must be adopted $^{25}$.

In this article, we shed a light onto the electronic properties of two-dimensional gold (2D-Au), by synthesizing it via the intercalation of $\mathrm{Au}$ atoms at the heterointerface between the Siterminated $\mathrm{SiC}(0001)$ surface and its C-rich $(6 \sqrt{3} \times 6 \sqrt{3}) \mathrm{R} 30^{\circ}$ reconstruction. $\mathrm{Au}$ atoms are arranged in a highly crystalline fashion and develop their own band dispersion, which is intrinsically two-dimensional. When a single layer of $\mathrm{Au}$ atoms is intercalated, a semiconductor (SC) crystal of hexagonal symmetry with the valence band maximum in $\overline{\mathrm{K}}_{\mathrm{Au}}$ close to the Fermi level and a saddle point in $\overline{\mathrm{M}}_{\mathrm{Au}}$ at about $400 \mathrm{meV}$ is formed. These $2 \mathrm{D}$ states also exhibit a significant spin-orbit splitting, amounting to $225 \mathrm{meV}$, along the $\overline{\Gamma K}$ dispersion direction. We show that the $2 \mathrm{D}$ semiconducting Au undergoes a transition to metal $(\mathrm{M})$ when the number of intercalated Au layers goes from one to two. Transition metal atoms have already been intercalated underneath the buffer layer graphene $\mathrm{e}^{26-28}$ or zerolayer graphene (ZLG) on $\mathrm{SiC}$ $(0001)$ as well as under epitaxial graphene on $\mathrm{Ni}(111)^{29}$. The effects reported were doping of the graphene $\pi$-bands $s^{30,31}$ or mini-gaps opening in the graphene Dirac cone due to moiré superperiodicity ${ }^{32}$. It was recently shown that the interaction between the $5 d$ orbitals of gold and the valence band of graphene can open a spin-orbit gap deep in the valence band of goldintercalated graphene on $\mathrm{SiC}(0001)^{33}$. However, no evidence of two-dimensional dispersing bands stemming from the metal layer was ever reported, which would be indicative of a well-defined order of the film.

\section{Results}

Properties of the 2D-Au semiconducting phase. Figure $1 \mathrm{a}, \mathrm{b}$ displays the band structure of the SC 2D-Au, measured by angleresolved photoemission spectroscopy (ARPES). Two spectral cuts are shown in Fig. 1a, which give the dispersion along the high symmetry directions $\overline{\Gamma K}_{\mathrm{Au}} \overline{\mathrm{M}}_{\mathrm{Au}}$ and $\bar{\Gamma}^{\prime} \overline{\mathrm{M}}_{\mathrm{Au}} \bar{\Gamma}^{\prime}$. From $\bar{\Gamma}$ towards $\overline{\mathrm{K}}_{\mathrm{Au}}$ the dispersion has a band velocity of $(1.00 \pm 0.05) \cdot 10^{6} \mathrm{~ms}^{-1}$ at a binding energy $-1 \mathrm{eV}$ for both the $\overline{\Gamma K}$ and $\overline{\Gamma M}$ directions, i.e., comparable with graphene's band velocity in the vicinity of the Fermi level. From $\overline{\mathrm{K}}_{\mathrm{Au}}$ towards $\overline{\mathrm{M}}_{\mathrm{Au}}$, the band is instead rather flat. At $\overline{\mathrm{K}}_{\mathrm{Au}}$ we identify the maximum of the electronic dispersion. In order to locate it with precision, we perform the sigmoidal fit of the energy dispersion curves cutting through the apical part of the graphene $\pi$-bands and the $\overline{\mathrm{K}}_{\mathrm{Au}}$ point. Such a fit with the respective energy locations is shown in Fig. $1 \mathrm{~d}$ and it tells us that the $2 \mathrm{D}$ gold valence band maximum (VBM) is located at -50 $\mathrm{meV}$. By performing a statistical analysis on the position of the VBM of 2D-Au for several samples, we find that the overall mean value falls just short of $-70 \mathrm{meV}$ (cf. Supplementary Note 1). The saddle point in $\overline{\mathrm{M}}_{\mathrm{Au}}$ is found at $-400 \mathrm{meV}$, meaning that the van Hove singularity (vHS) in the density of states is at an energy at reach for electronic measurements. No distinct crossing of the Fermi level is observed, in line with the missing Fermi surface contour in Fig. 1c. We observe a faint triangularly shaped spectral weight at the Fermi level, due to the intrinsic width of the band. By connecting the vertexes of the aforementioned intensities, we define the Brillouin zone (BZ) of the SC 2D-Au, drawn as a blue hexagon in Fig. 1c.

Photon energy dependent ARPES measurements were performed to probe a possible crystallographic periodicity along the $\hat{z}$ axis (see Supplementary Note 2), and confirmed the twodimensional nature of the gold layer. Furthermore, a highresolution close-up of the bands along the $\overline{\Gamma K}_{\mathrm{Au}}$ direction shows that the states exhibit a spin-splitting which reaches the maximum value of $225 \mathrm{meV}$ at $-0.5 \mathrm{eV}$. It should be mentioned that since the states observed in this energy range and in this $k$ space portion have no correspondence in any other graphene/SiC (0001) based system $26,32,34$ they can solely be attributed to the interfacial $\mathrm{Au}$ layer.

The gold atoms at the interface affect the electronic properties of graphene ${ }^{31}$. A single gold layer acts as an electron donor to graphene, shifting the $\pi$-bands down until the Dirac point $E_{0}$ is observed about $700 \mathrm{meV}$ under the Fermi level, as visible from Fig. 1e, where we show an ARPES cut through graphene's $\overline{\mathrm{K}}_{\mathrm{Gr}}$ point, taken perpendicular to the $\overline{\Gamma K}_{\mathrm{Gr}}$ direction. In addition, the overall dielectric constant of the system is such that many-particle effects emerge and can be detected (cf. Supplementary Figs. 3 and 4). In particular, we extract an effective dielectric constant of $\varepsilon_{\text {eff }}$ $=7 \pm 1$, which allow us to detect the plasmaron band ${ }^{35}$. The crossing energy of the plasmaron band is indicated as $E_{1}$ and further details can be found in the Supplementary Note 3.

The size and shape of the 2D-Au BZ suggest that the Au atoms are arranged on a triangular lattice with the lattice parameter matching that of $\mathrm{SiC}(0001)$, i.e., $3.08 \AA$. As a further support to this hypothesis, we have observed with ARPES, graphene replica bands shifted by a $\mathrm{SiC}(0001)$ reciprocal lattice vector (cf. Supplementary Note 4).

We compared the observed bands to a tight binding (TB) model calculated on this $2 \mathrm{D}$ triangular lattice in the next-nearest neighbor (NNN) approximation:

$$
\begin{aligned}
g(\vec{k}) & =-\varepsilon_{0}-\gamma_{01} u_{1}(\vec{k})-\gamma_{02} u_{2}(\vec{k}) \\
u_{1}(\vec{k}) & =2 \cos \left(k_{x} a\right)+4 \cos \left(k_{x} a / 2\right) \cos \left(k_{y} \sqrt{3} a / 2\right) \\
u_{2}(\vec{k}) & =2 \cos \left(k_{y} \sqrt{3} a\right)+4 \cos \left(k_{y} \sqrt{3} a / 2\right) \cos \left(k_{x} 3 a / 2\right)
\end{aligned}
$$

where $a$ is the lattice parameter, $\gamma_{01}$ and $\gamma_{02}$ are the NN and NNN hopping parameters, the first being of the order of $1 \mathrm{eV}$ and $\gamma_{02} \simeq \gamma_{01} / 10$. For the plot of Fig. $1 \mathrm{a}$, b we set $\gamma_{01}=1.1 \mathrm{eV}$ and $a=$ $3.08 \AA$, that is the $\mathrm{SiC}(0001)$ lattice parameter.

Apparently, even this simple model describes the measured band dispersion quite well. In particular, the extremes are placed at the same $k$, confirming the right choice of $a$. The validity of the model also confirms the $2 \mathrm{D}$ nature of the states. However, some small deviations are present, especially around $\overline{\mathrm{M}}_{\mathrm{Au}}$.

Density functional theory (DFT) calculations have been published for this system by Chuang et al. ${ }^{36}$. These simulations show the formation of Au-interface related bands, similar to the 

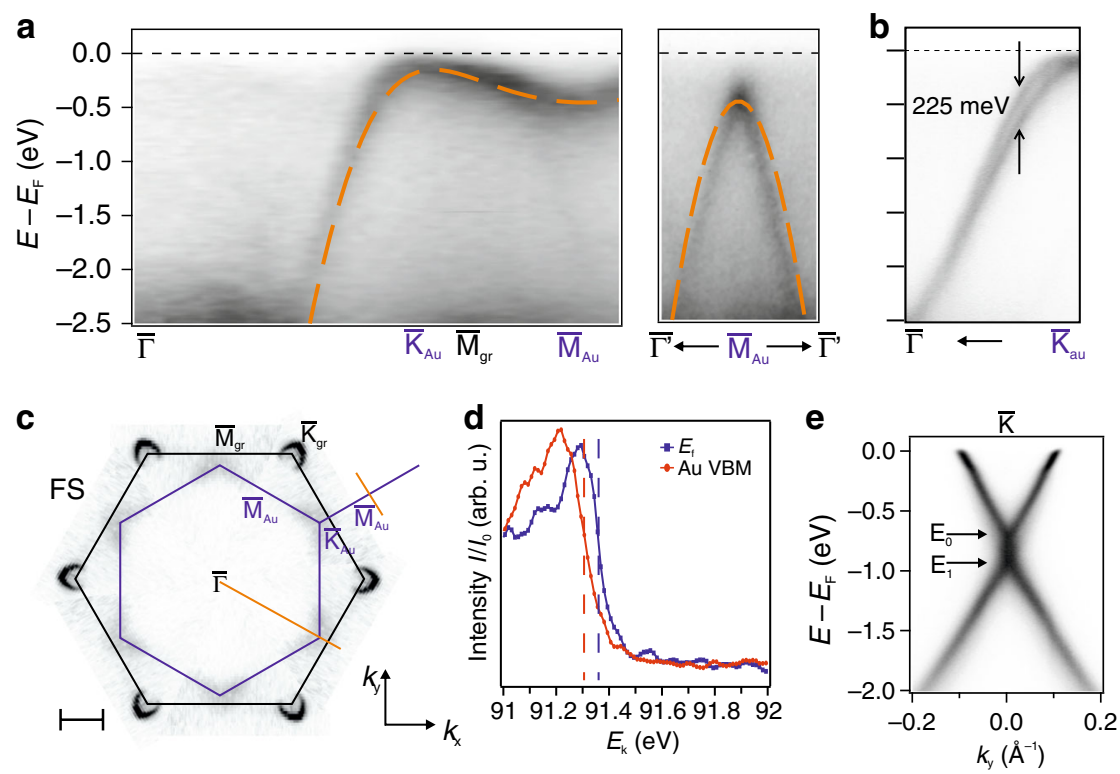

Fig. 1 Electronic properties of 2D Gold. a ARPES cut along the diagonal orange line in panel c, showing only the Au bands dispersion. In the right panel the spectrum cuts along the short orange line in panel $\mathbf{c}$. The orange dashed lines superimposed to the data are the TB bands as described in the text. $\mathbf{b}$ Au bands dispersion along the $k_{y}$ direction highlighting their spin-orbit splitting $\Delta S O$. The photon energy for panels $\mathbf{a}$ and $\mathbf{b}$ is $95 \mathrm{eV}$. c Fermi surface of the system measured by ARPES. The figure is the result of the sixfold symmetrisation of a measurement. Scale-bar corresponding to $0.5 \AA^{-1}$. $\mathbf{d}$ Normalized line profiles extracted along the graphene $\pi$-band and the $\overline{\mathrm{K}}_{\mathrm{Au}}$ point (cf. Supplementary Fig. 1). e Dispersion measured along $k_{y}$ at the $\overline{\mathrm{K}}_{\mathrm{Gr}}$ point with $40 \mathrm{eV}$ photons, showing graphene's Dirac cone.

ones observed in our experiment. There, it was presumed, that the structure of the interfacial $\mathrm{Au}$ is arranged such that every topmost $\mathrm{Si}$ atom of the $\mathrm{SiC}$ is bound to one $\mathrm{Au}$ atom, i.e. a $(1 \times 1)$ with respect to the $\mathrm{SiC}(0001)$. Similar to our experiment, their bands have the maximum in close proximity to the Fermi level, which indicates a semiconducting behavior.

Moreover, in ref. 37 the authors find a strong spin-splitting stemming from spin-orbit coupling. The spin texture there resembles a Rashba-type with a Rashba parameter of $1.638 \mathrm{eV} \AA$ ${ }^{-1}$. In fact, we do find a strong correspondence to this in the experimental band structure (see Fig. 1b). Extracting the Rashba parameter from the experiments, we find a value of $2.17 \mathrm{eV} \AA^{-1}$, which is in rather good agreement with theory.

In order to gather further information about the actual structure of the interfacial gold layer, we carried out X-ray photoemission spectroscopy (XPS), low-energy electron diffraction (LEED) and scanning tunneling microscopy (STM) measurements. Representative spectra of the $\mathrm{Au} 4 f$ and $\mathrm{Si} 2 p$ core levels are shown in Fig. 2a, b, respectively.

The details about the fitting parameters are reported in Supplementary Table 1 . The Au $4 f$ doublet indicates the presence of atoms in a single chemical environment. The binding energy that we measure is $\sim 350 \mathrm{meV}$ higher than for metallic gold and this excludes the presence of a substantial amount of metallic gold either on the surface or underneath graphene. In contrast, it indicates that $\mathrm{Au}$ is bound to $\mathrm{Si}$ at the interface, as confirmed by the Si $2 p$ spectrum, which shows a component of bulk SiC and a surface component of Au-Si. Symmetry considerations allow us to conclude that, since the bands we observe are of $s$ - $p$ character, the $\mathrm{Au}$ atoms must interact with the $2 p \mathrm{Si}$ orbitals by their $5 d$ electrons.

It should be noted that this does not represent an alloyed silicide layer, which is also reported to have a different (higher) binding energy ${ }^{38}$. What we observe is rather a significant electronic overlap between the topmost $\mathrm{Si}$ atoms of the substrate
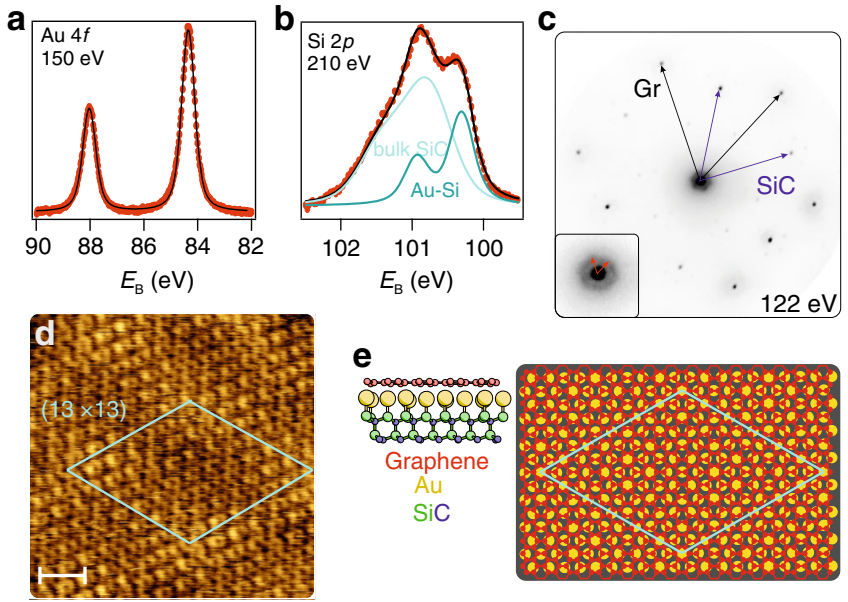

Fig. 2 Structural properties of the interface. Core level spectra acquired on a Au-intercalated monolayer of epitaxial graphene on $\mathrm{SiC}(0001)$ of a $\mathrm{Au} 4 f$ fitted with a single Voigt doublet, $\mathbf{b}$ Si $2 p$ fitted with two Voigt doublets. The photon energies are indicated in the panels. $\mathbf{c} \mu$ LEED pattern recorded at $122 \mathrm{eV}$ on Au-intercalated graphene. The inset highlights the $(13 \times 13)$ pattern observed around the (00) spot. d STM topography image over a $(5.2 \times 5.2) \mathrm{nm}^{2}$ region. The $(13 \times 13)$ moiré unit cell is indicated as a cyan diamond. Image recorded at $U_{\text {sample }}=100 \mathrm{mV}$ and $I_{\text {tunnel }}=85 \mathrm{pA}$. Scalebar $1 \mathrm{~nm}$. e Ball-and-stick model of the gold atoms intercalated between graphene and $\mathrm{SiC}(0001)$. Side view and, on the right side, top view showing the $(13 \times 13)$ supercell.

and the intercalated gold layer. The interaction with the substrate stabilizes the gold layer and induces the interfacial order. Notably, $\mathrm{Au}$ atoms deposited onto $\mathrm{SiC}(0001)$ are observed not to order on the $(1 \times 1)^{38,39}$, which implies the need for the graphene on top to impose the observed ordering and electronic structure. 


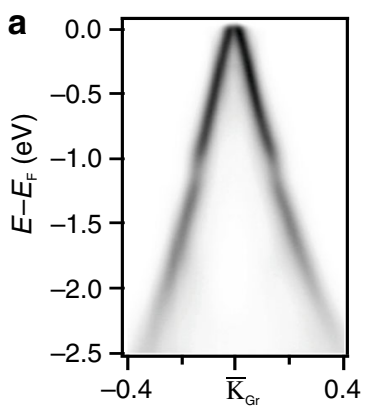

d

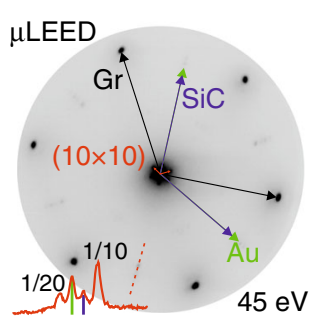

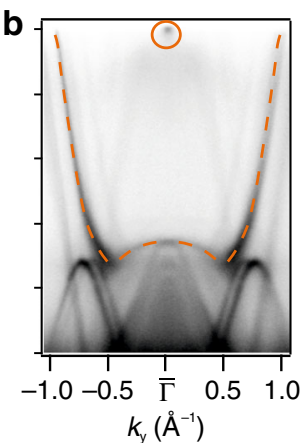

e

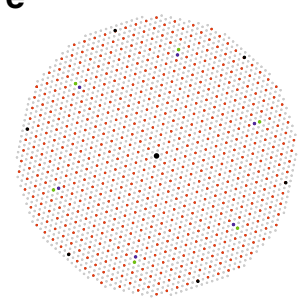

C
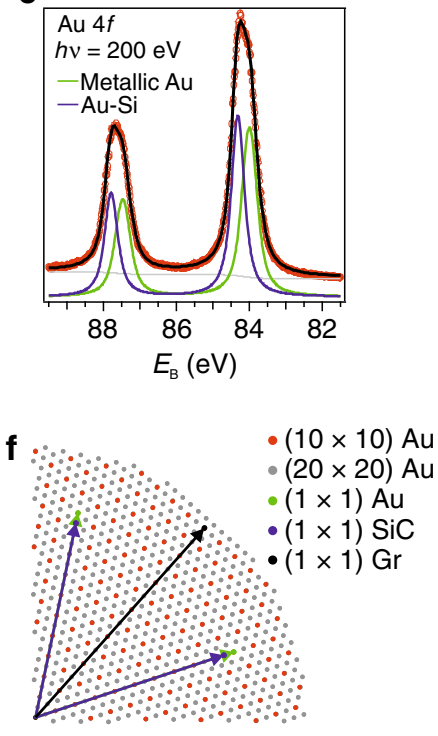

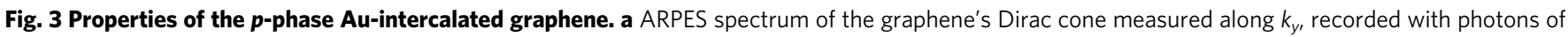
$40 \mathrm{eV}$. b ARPES spectrum measured at $75 \mathrm{eV}$ centered in $\bar{\Gamma}$, showing the band structure of the intercalated gold along the $k_{y}$ direction. c $\mu$ XPS spectrum of Au $4 f$ doublet. $\mathbf{d} \mu$ LEED pattern of the system. In the bottom-left corner the line profile along the red line is shown, highlighting the four diffraction spots visible and assigning them to SiC (blue), Au (green) and the 1/20 and 1/10 Au superperiodicities. e Sketch of the $\mu$ LEED with all the periodicities indicated. $\mathbf{f}$ Blow-up of one quadrant of panel $\mathbf{e}$.

The $\mu$-LEED pattern of Fig. $2 c$ shows that after the intercalation of $\mathrm{Au}$ atoms, only the graphene and $\mathrm{SiC}(1 \times 1)$ are visible. In addition, a hexagonal halo oriented like graphene and $1 / 13$ of its dimension is discernible. Atomically resolved STM carried out on such a sample, further corroborates the atomic arrangement. The STM image in Fig. 2d shows a region where the $(13 \times 13)$ superpotential is well visible and the graphene lattice is atomically resolved (see also Supplementary Fig. 9).

From the data reported above, we find that every Au atom is bound to a $\mathrm{Si}$ atom at the interface (cf. Supplementary Fig. 7), meaning that the $\mathrm{Au}$ is ordered in a $(1 \times 1)$ registry with respect to the $\mathrm{SiC}$, as it was found from ARPES. In Fig. 2e we therefore report a ball-and-stick model of the interface in side and top view, to better illustrate the mutual arrangement between gold and graphene.

Properties of the 2D-Au metallic phase. By extending the gold deposition time, it is possible to intercalate a double layer of gold (see Methods for more details). Going from a single Au layer to a Au bilayer does not only switch the doping of graphene from $n+$ to slightly $p$, but it also switches the character of the $2 \mathrm{D}-\mathrm{Au}$ electronic properties. In fact, we observe a transition from SC to a $\mathrm{M}$ behavior.

Figure 3 summarizes the electronic, chemical and structural properties of the M 2D-Au. In the first place, the graphene - shown in panel $\mathrm{a}-$ is marginally hole-doped with a density of states of $(9 \pm$ $1) \cdot 10^{11} \mathrm{~cm}^{-2}$, which, given a band velocity of $(1.33 \pm 0.05) \cdot 10^{6} \mathrm{~ms}^{-1}$, corresponds to a shift of the Fermi level by $(-150 \pm 10) \mathrm{meV}$ with respect to the Dirac point. We as well observe the Rashba splitting at about $-1 \mathrm{eV}$ due to the interaction between the graphene and gold bands, as reported in ref. ${ }^{33}$.

The interfacial gold still develops its own band dispersion, with a rich structure, as exemplary shown in Fig. 3b, where we show an ARPES spectrum recorded at $75 \mathrm{eV}$ and centered in $\bar{\Gamma}$ along $k_{y}$ direction, with reference to Fig. 1c. The downward parabolic states centered in $\bar{\Gamma}$ are gold quantum well states, as previously observed for very thin gold films on metal ${ }^{24}$. Similar states are as well observed for the SC phase, as visible from the ARPES data acquired at 75 and $40 \mathrm{eV}$, reported in Supplementary Figs. 2 and 5, respectively.

We mark the states reaching the Fermi level with the orange dashed line and a solid circle. The spectral weight at $\bar{\Gamma}$ at the Fermi level represents the bottom of a conduction band of the M 2D-Au and it is not related with the Shockley state of the $\mathrm{Au}(111)$ surface (cf. Supplementary Fig. 10). It was predicted in previously published calculations ${ }^{36}$, even if they considered three layers at the interface instead of two. Such a band structure implies that at any given small variation of the chemical potential, or applied external voltage, there will always be a finite electronic density of states, confirming the metallicity of this phase.

XPS measurements confirm the presence of a gold double layer, as does STM (see Supplementary Note 6). Figure 3c displays the $\mathrm{Au} 4 f$ doublet fitted with two components. The ratio between the two is very close to one, indicating a symmetric distribution of the atoms between the first and the second layer.

Due to the favored interatomic forces, the intercalated gold bilayer shrinks its lattice parameter with respect to the monolayer. The gold atoms then arrange in a configuration closer to the bulk $\mathrm{Au}(111)$. This is illustrated by the $\mu$-LEED data shown in Fig. $3 \mathrm{~d}$. A hexagonal pattern around the (00) spot is well visible. That vector is $1 / 10$ of the $\mathrm{Au}$ reciprocal lattice vector. Yet, even a denser grid is visible, as the line profile (enlarged to be better seen) in the bottom-left part of the panel indicates.

Such a grid-represented in Fig. 3e, f-emerges from the $\mathrm{Au} /$ $\mathrm{SiC}(0001)$ interface and it corresponds to $\mathrm{Au}$ atoms being arranged as $(20 \times 20)$ over $(19 \times 19) \quad \mathrm{SiC}$ unit cells. The corresponding lattice parameter for the gold M-phase is extracted from several different LEED, $\mu$ LEED, ARPES, and STM measurements. The estimation of the lattice parameter is $(2.93 \pm 0.01) \AA$, about $1.6 \%$ larger than the nominal $2.883 \AA$ of the $\mathrm{Au}(111)$.

When such a gold bilayer interacts with graphene, it develops a superperiodicity half the size, which is observed in STM, as visible in the data reported in Fig. 4. The Gr/Au moiré is well identified in the topographical STM measurements of Fig. 4a. The graphene lattice is very well resolved as well and the superlattice unit cell is 

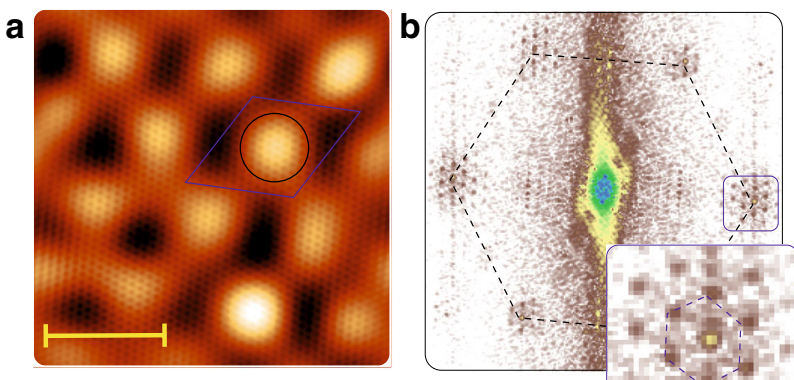

\section{C \\ BZs}

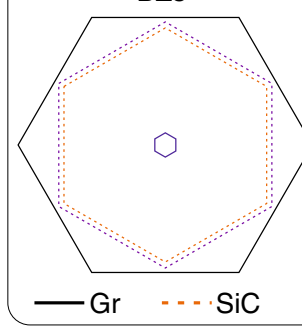

Real space

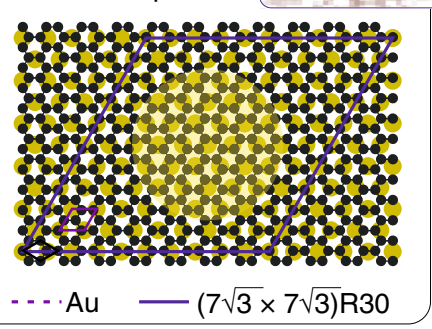

Fig. 4 Moiré structure of $\mathbf{M}$ 2D-Au. a STM image 2D-FFT filtered $U_{\text {sample }}=$ $-105 \mathrm{mV} I_{\text {tunnel }}=325 \mathrm{pA}$. Scale-bar $3 \mathrm{~nm}$. b 2D-FFT of the real-space image in panel $\mathbf{a}$. In the inset: zoom-in with respect to the blue box in panel $\mathbf{b}$. c Reciprocal and real-space arrangement between graphene and gold. All BZs are $90^{\circ}$ rotated in order to match the alignment used for the ARPES description.

drawn in the image as a blue diamond. In Fig. $4 \mathrm{~b}$, the fast $2 \mathrm{D}$ Fourier transformation (2D-FFT) of the image (raw data in Supplementary Fig. 7) shows distinctively the graphene and superstructure periodicities and helps us conclude that $(7 \sqrt{3} \times$ $7 \sqrt{3})$ R30 graphene unit cells are arranged on $(10 \times 10)$ of gold, which is the strongest superperiodicity observed also in LEED (cf. Supplementary Fig. 8). The proposed model for the atomic arrangement of graphene and M 2D-Au is shown in Fig. 4c. The region within the supercell where the $\mathrm{C}$-Au distance is larger is circled in panel a and indicated as a yellow disc in panel $\mathbf{c}$. The difference in $\mathrm{C}$-Au adsorption distance emerges from the local variation of the atomic registry between the lattices.

\section{Discussion}

The intercalation method presented in this work yields a van der Waals heterostructure between a 2D semimetal, i.e., graphene and a $2 \mathrm{D}$ material formed by a triangular lattice of gold atoms, the electronic properties of which can be tuned, depending on the number of layers. By synthesizing a single layer of gold at the heterointerface between graphene and $6 \mathrm{H}-\mathrm{SiC}(0001)$, we define a material with semiconductor character, exhibiting the maximum of the valence band at an average energy of $67 \mathrm{meV}$ below the Fermi level. We have determined that the gold atoms arrange themselves in a highly ordered triangular lattice, mimicking the $\mathrm{SiC}(1 \times 1)$ and develop their own band structure. Such an electronic band dispersion has a truly two-dimensional character and it is notably well reproduced by a simple TB model. Tuning the amount of intercalated gold from one to two layers, leads to a two-dimensional gold with metallic character. The interatomic forces within the gold layer make the lattice parameter shrink from $3.08 \AA$, to $2.93 \AA$, yet not quite relaxed into the $\mathrm{Au}(111)$ configuration, which has $2.88 \AA$ as atomic spacing. Graphene is also affected by the underlying $2 \mathrm{D}$ gold in terms of electronic properties. Its doping level goes from $n=(3.45 \pm 0.05) \cdot 10^{13} \mathrm{~cm}^{-2}$ when on SC $2 \mathrm{D}-\mathrm{Au}$, to $p=(9 \pm 1) \cdot 10^{11} \mathrm{~cm}^{-2}$ when on M $2 \mathrm{D}-\mathrm{Au}$. The doping difference, i.e., a shift in the Dirac point position, is about $700 \mathrm{meV}$ and is reflected almost entirely to a work function difference between the two phases (see Supplementary Fig. 6). The system therefore results in a double lateral junction: the graphene exhibit a $p / n^{+}$junction, in correspondence to the M/SC transition region of the $2 \mathrm{D}-\mathrm{Au}$, which represents a lateral Schottky contact. In addition, SC 2D-Au exhibits a vHS at about $400 \mathrm{meV}$ from the Fermi edge, making this system a very appealing platform for the observation of collective phenomena at low temperatures. The whole structure is monolithic and does not require any transfer.

Besides the deep fundamental interest provided by this class of materials, we envision a vast portfolio of possible applications for this system, ranging from detection of $\mathrm{THz}$ radiation, to more sophisticated optoelectronic devices involving the spin degree of freedom. It will moreover serve as a platform for exploring nonlinear optics effects in two-dimensional gold, a field so far essentially unexplored.

\section{Methods}

Sample preparation. Nominally on-axis oriented single crystalline single-side polished $6 \mathrm{H}$-SiC $n$-type substrates were used in the present study and were purchased from SiCrystal GmbH. Polishing damages were removed by etching the substrate at $1500{ }^{\circ} \mathrm{C}$ in a purified $\mathrm{H}_{2}$ atmosphere. The $\mathrm{SiC}(0001)$ surfaces were graphitized by annealing the samples in argon atmosphere following the procedure introduced by Emtsev et al. in ref. ${ }^{40}$. The ZLG develops upon annealing in $\mathrm{Ar}$ atmosphere at about $1400{ }^{\circ} \mathrm{C}$ for $10 \mathrm{~min}^{41-45}$. The formation of the ZLG results in a carbon-rich reconstruction with periodicity $(6 \sqrt{3} \times 6 \sqrt{3})$ $\mathrm{R} 30^{\circ}$ with respect to the $\mathrm{SiC}(0001)$ unit cell ${ }^{26-28[, 46}$. The samples were prepared by evaporating atomic Au from a Knudsen cell onto the ZLG surface, keeping the sample at temperatures between 600 and $700{ }^{\circ} \mathrm{C}$ and by subsequent annealing of the sample at temperatures between 800 and $850^{\circ} \mathrm{C}$. Keeping the sample at that temperature should favor the intercalation process over the diffusion of $\mathrm{Au}$ atoms over the surface and the consequent formation of clusters. $\mathrm{Au}$ is known to have a very high mobility on graphene ${ }^{47}$ so that deposition at room temperature followed by annealing leads to cluster formation ${ }^{31}$. Preparing the samples in the way described here helps in keeping the surface more clean. The single-layer gold is prepared in the aforementioned way by depositing a nominal amount of about 20 gold monolayers at a rate of $5 \AA$ per minute. In this way the deposition takes about $10 \mathrm{~min}$. For the gold bilayer a deposition time ranging from 20 to $25 \mathrm{~min}$ is used, employing the same rate. We point out that the intercalated samples are very stable in air ( $\tau \gg$ months) and that they do not require any particular protection from the atmospheric environment. In UHV conditions, they can be heated up to $850{ }^{\circ} \mathrm{C}$ before the intercalated gold starts to be deintercalated and desorbed from the surface.

Measurements. The band structure of the system was mapped by means of ARPES at the end-station $1^{2}$ of the BESSY II (Berlin, Germany) synchrotron facility at a temperature of $90 \mathrm{~K}$. The hemispherical electron analyzer used for the data harvesting was a Scienta R8000 with a diameter of $200 \mathrm{~mm}$ and an ultimate energy resolution of $1 \mathrm{meV}$. In our experiments the instrumental resolution was set to about $2.5 \mathrm{meV}$, hence smaller than the thermal broadening of the bands, i.e., about $7.5 \mathrm{meV}$. Additional ARPES measurements have been carried out at the I4 endstation of the MAXlab (Lund, Sweden) synchrotron facility with a SPECS Phoibos 100 analyzer. The aforementioned lines have also been used to acquire X-ray photoelectron spectroscopy (XPS) data. Preliminary studies to clarify the preparation procedures and to assess the sample's quality were carried out in the home-laboratory at the MPI Stuttgart using a Specs Phoibos 150 analyzer and monochromatized He II radiation for the photoexcitation. Low-energy electron microscopy (LEEM) measurements, as well as micro-ARPES ( $\mu$ ARPES), microLEED ( $\mu$ LEED), and micro-XPS, were acquired at the beamline I311 beamline of the Maxlab using an Elmitec III microscope. More details about the performances of the microscope can be found elsewhere ${ }^{28,48}$. The scanning tunneling microscopy (STM) measurements were performed with an Omicron LT-STM microscope at the CNI@NEST in Pisa at a temperature of $78 \mathrm{~K}$.

\section{Data availability}

The authors declare that the main data supporting the findings of this study are available within the article and its Supplementary Information files. Extra data are available from the corresponding author upon request.

Received: 19 April 2019; Accepted: 23 March 2020; Published online: 06 May 2020 


\section{References}

1. Berger, C. et al. Ultrathin epitaxial graphite: $2 \mathrm{D}$ electron gas properties and a route toward graphene-based nanoelectronics. J. Phys. Chem. B 108, 19912 (2004).

2. Novoselov, K. S. et al. Electric field effect in atomically thin carbon films. Science 306, 666 (2004).

3. Nagashima, A., Tejima, N., Gamou, Y., Kawai, T. \& Oshima, C. Electronic structure of monolayer hexagonal boron nitride physisorbed on metal surfaces. Phys. Rev. Lett. 75, 3918 (1995).

4. Lv, R. et al. Transition metal dichalcogenides and beyond: synthesis, properties, and applications of single- and few-layer nanosheets. Acc. Chem. Res. 48, 56-64 (2015).

5. Lalmi, B. et al. Epitaxial growth of a silicene sheet. Appl. Phys. Lett. 97, 223109 (2010).

6. Dávila, M. E., Xian, L., Cahangirov, S., Rubio, A. \& Le Lay, G. Germanene: a novel two-dimensional germanium allotrope akin to graphene and silicene. $N$. J. Phys. 16, 095002 (2014).

7. Bampoulis, P. et al. Germanene termination of $\mathrm{Ge}_{2} \mathrm{Pt}$ crystals on $\mathrm{Ge}(110) . J$. Phys. Condens. Mat. 26, 442001 (2014).

8. Zhu, F. F. et al. Epitaxial growth of two-dimensional stanene. Nat. Mater. 14 1020-1025 (2015).

9. Ji, J. et al. Two-dimensional antimonene single crystals grown by van der Waals epitaxy. Nat. Commun. 7, 1-9 (2016).

10. Reis, F. et al. Bismuthene on a SiC substrate: a candidate for a hightemperature quantum spin Hall material. Science 357, 287-290 (2017).

11. Li, L. et al. Two-dimensional transition metal honeycomb realized: Hf on $\mathrm{Ir}$ (111). Nano Lett. 13, 4671-4674 (2013).

12. Duan, H. et al. Ultrathin rhodium nanosheets. Nat. Commun. 5, 1-8 (2014).

13. Zhao, J. et al. Free-standing single-atom-thick iron membranes suspended in graphene pores. Science 343, 1228-1232 (2014).

14. Mounet, N. et al. Two-dimensional materials from high-throughput computational exfoliation of experimentally known compounds. Nat. Nanotechnol. 13, 246-252 (2018).

15. Chen, Y. et al. Two-dimensional metal nanomaterials: synthesis, properties, and applications. Chem. Rev. 118, 6409-6455 (2018).

16. Sinibaldi, A. et al. Direct comparison of the performance of Bloch surface wave and surface plasmon polariton sensors. Sens. Actuators, B: Chem. 174, 292-298 (2012).

17. Freeman, A. J. \& Fu, C. L. Strongly enhanced 2D magnetism at surfaces and interfaces (invited). J. Appl. Phys. 61, 3356-3361 (1987).

18. Yafet, Y., Kwo, J. \& Gyorgy, E. M. Dipole-dipole interactions and twodimensional magnetism. Phys. Rev. B 33, 6519-6522 (1986).

19. Siegmann, H. C. Surface and 2D magnetism. J. Phys.: Condens. Matter 4 8395-8434 (1992).

20. Gibertini, M., Koperski, M., Morpurgo, A. F. \& Novoselov, K. S. Magnetic 2D materials and heterostructures. Nat. Nanotechnol. 14, 408-419 (2019).

21. Jacobsen, J. et al. Atomic-scale determination of misfit dislocation loops at metal-metal interfaces. Phys. Rev. Lett. 75, 489-492 (1995).

22. Shikin, A. M. et al. Origin of spin-orbit splitting for monolayers of $\mathrm{Au}$ and $\mathrm{Ag}$ on W(110) and Mo(110). Phys. Rev. Lett. 100, 1-4 (2008).

23. Shikin, A. M. et al. Induced Rashba splitting of electronic states in monolayers of $\mathrm{Au}, \mathrm{Cu}$ on a W(110) substrate. N. J. Phys. 15, 095005 (2013).

24. Varykhalov, A. et al. Quantum cavity for Spin due to spin-orbit interaction at a metal boundary. Phys. Rev. Lett. 101, 256601 (2008).

25. Lee, J., Yang, J., Kwon, S. G. \& Hyeon, T. Nonclassical nucleation and growth of inorganic nanoparticles. Nat. Rev. Mater. 1, 16034 (2016).

26. Emtsev, K. V., Speck, F., Seyller, T., Ley, L. \& Riley, J. D. Interaction, growth, and ordering of epitaxial graphene on $\mathrm{SiC}\{0001\}$ surfaces: A comparative photoelectron spectroscopy study. Phys. Rev. B 77, 155303 (2008).

27. Riedl, C., Coletti, C. \& Starke, U. Structural and electronic properties of epitaxial graphene on $\mathrm{SiC}(0001)$ : a review of growth, characterization, transfer doping and hydrogen intercalation. J. Phys. D Appl. Phys. 43, 374009 (2010).

28. Forti, S. \& Starke, U. Epitaxial graphene on SiC: from carrier density engineering to quasi-free standing graphene by atomic intercalation. J. Phys. D Appl. Phys. 47, 094013 (2014).

29. Varykhalov, A. et al. Electronic and magnetic properties of quasifreestanding graphene on Ni. Phys. Rev. Lett. 101, 157601 (2008).

30. Gierz, I., Riedl, C., Starke, U., Ast, C. R. \& Kern, K. Atomic hole doping of graphene. Nano Lett. 8, 4603 (2008).

31. Gierz, I. et al. Electronic decoupling of an epitaxial graphene monolayer by gold intercalation. Phys. Rev. B 81, 235408 (2010).

32. Forti, S. et al. Mini-Dirac cones in the band structure of a copper intercalated epitaxial graphene superlattice. 2D Mater. 3, 035003 (2016).

33. Marchenko, D., Varykhalov, A., Sánchez-Barriga, J., Seyller, T. \& Rader, O. Rashba splitting of $100 \mathrm{meV}$ in Au-intercalated graphene on SiC. Appl. Phys. Lett. 108, 172405 (2016).

34. Bostwick, A., Ohta, T., Seyller, T., Horn, K. \& Rotenberg, E. Quasiparticle dynamics in graphene. Nat. Phys. 3, 36 (2007).
35. Bostwick, A. et al. Observation of plasmarons in Quasi-freestanding doped graphene. Science 328, 999 (2010).

36. Chuang, F.-C. et al. Electronic structures of an epitaxial graphene monolayer on $\mathrm{SiC}(0001)$ after gold intercalation: a first-principles study. Nanotechnology 22, 275704 (2011)

37. Hsu, C. H., Lin, W. H., Ozolins, V. \& Chuang, F. C. Electronic structures of an epitaxial graphene monolayer on $\mathrm{SiC}(0001)$ after metal intercalation (metal $=\mathrm{Al}$ Ag, Au, Pt, and Pd): A first-principles study. Appl. Phys. Lett. 100, 063115 (2012).

38. Virojanadara, C. \& Johansson, L. I. Electronic structure of reconstructed AuSiC(0001) surfaces. Surf. Sci. 600, 436 (2006).

39. Stoltz, D., Stoltz, S. E. \& Johansson, L. S. O. A high-resolution core-level photoemission study of the $\mathrm{Au} / 4 \mathrm{H}-\mathrm{SiC}(0001)-(\sqrt{3} \times \sqrt{3})$ interface. J. Phys. Cond. Matt 19, 266006 (2007)

40. Emtsev, K. V. et al. Towards wafer-size graphene layers by atmospheric pressure graphitization of silicon carbide. Nat. Mat. 8, 203 (2009).

41. van Bommel, A. J., Crombeen, J. E. \& van Tooren, A. LEED and Auge electron observations of the $\operatorname{SiC}(0001)$ surface. Surf. Sci. 48, 463 (1975).

42. Forbeaux, I., Themlin, J.-M. \& Debever, J.-M. Heteroepitaxial graphite on $6 \mathrm{H}$ $\mathrm{SiC}(0001)$ :Interface formation through conduction-band electronic structure. Phys. Rev. B 58, 16396 (1998)

43. Mattausch, A. \& Pankratov, O. Ab initio study of graphene on SiC. Phys. Rev. Lett. 99, 076802 (2007)

44. Varchon, F. et al. Electronic structure of epitaxial graphene layers on SiC: effect of the substrate. Phys. Rev. Lett. 99, 126805 (2007).

45. Riedl, C., Starke, U., Bernhardt, J., Franke, M. \& Heinz, K. Structural properties of the graphene-SiC(0001) interface as a key for the preparation of homogeneous large-terrace graphene surfaces. Phys. Rev. B 76, 245406 (2007)

46. Starke, U., Forti, S., Emtsev, K. \& Coletti, C. Engineering the electronic structure of epitaxial graphene by transfer doping and atomic intercalation. MRS Bull. 37, 1177 (2012).

47. Liu, X. et al. Metals on graphene: interactions, growth morphology, and thermal stability. Crystals 3, 79-111 (2013).

48. Forti, S. et al. Large-area homogeneous quasifree standing epitaxial graphene on $\mathrm{SiC}(0001)$ : Electronic and structural characterization. Phys. Rev. B 84, 125449 (2011).

\section{Acknowledgements}

This work was supported by the Deutsche Forschungsgemeinschaft in the framework of the Priority Program 1459 Graphene (Sta315/8-2). This research was partially funded by the European Community's Seventh Framework Programme: Research Infrastructures (FP7/2007-2013) under grant agreement no. 226716. The research leading to these results has received funding from the EuropeanUnion's Horizon 2020 Research and Innovation Program under grant agreement No. 785219GrapheneCore2. A.A.Z. would like to acknowledge support from Siftelsen för Strategisk Forskning (project RMA15-0024). A special thank goes to Thiagarajan Balasubramanian for his invaluable help for the acquisition of the ARPES spectra at the I4 beamline of the MAXlab. We thank HZB (Berlin, Germany) as well as MAX-lab (Lund, Sweden) for the allocation of synchrotron radiation beamtime. Support by the staff at Bessy (HZB) and MAX-Lab is gratefully acknowledged.

\section{Author contributions}

S.F. designed the experiments, grew the samples, performed ARPES, XPS and STM measurements, carried out the analysis of the data, and wrote the manuscript. S.L. and A S. helped with the measurements, the data analysis and the writing of the manuscript. Y N. and A.Z. carried out LEEM/PEEM measurements. C.C. participated in and supervised the STM measurements. U.S. participated in the synchrotron measurements and codesigned the experiments. C.C and U.S. supervised the project. All authors edited and commented the manuscript.

\section{Competing interests}

The authors declare no competing interests.

\section{Additional information}

Supplementary information is available for this paper at https://doi.org/10.1038/s41467 020-15683-1.

Correspondence and requests for materials should be addressed to S.F. or C.C.

Peer review information Nature Communications thanks the anonymous reviewer(s) for their contribution to the peer review of this work

Reprints and permission information is available at http://www.nature.com/reprints

Publisher's note Springer Nature remains neutral with regard to jurisdictional claims in published maps and institutional affiliations. 
(c) (i) Open Access This article is licensed under a Creative Commons Attribution 4.0 International License, which permits use, sharing, adaptation, distribution and reproduction in any medium or format, as long as you give appropriate credit to the original author(s) and the source, provide a link to the Creative Commons license, and indicate if changes were made. The images or other third party material in this article are included in the article's Creative Commons license, unless indicated otherwise in a credit line to the material. If material is not included in the article's Creative Commons license and your intended use is not permitted by statutory regulation or exceeds the permitted use, you will need to obtain permission directly from the copyright holder. To view a copy of this license, visit http://creativecommons.org/ licenses/by/4.0/.

(C) The Author(s) 2020 